\title{
Head and body stabilization in blowflies walking on differently structured substrates
}

\author{
Daniel Kress* and Martin Egelhaaf \\ Department of Neurobiology and CITEC Center of Excellence Cognitive Interaction Technology, Bielefeld University, \\ Universitätsstraße 25, 33615 Bielefeld, Germany \\ *Author for correspondence (daniel.kress@uni-bielefeld.de)
}

Accepted 14 January 2012

\begin{abstract}
SUMMARY
Visually guided animals depend heavily on the quality of visual signals in order to obtain functionally relevant information about their environment. To support visual information processing, nature has evolved a large variety of physiological adaptations and behavioral strategies such as compensatory head movements. During self-movement, head rotations compensate for changes in body attitude in order to stabilize gaze. However, how walking animals cope with uneven structured substrates, which may affect body and gaze orientation, is still unknown. We used stereo high-speed video to analyze compensatory head movements of blowflies walking freely on differently structured substrates. We found that even a pronounced asperity of the ground structure, with bumps of almost the size of the animal, was largely compensated by the walking apparatus of the blowfly, which leads to body roll and pitch movements only marginally larger than those on flat substrate. Pitch and roll fluctuations of the head were smaller compared with body fluctuations on all tested substrates, emphasizing the significance of gaze stabilization during walking on structured substrates. Furthermore, we found no impairment in head and body stabilization during walks in darkness, which indicates that the control system mediating compensatory head movements works well without any visual input. Interestingly, blowflies changed their walking style in the dark and seemed to use their forelegs as tactile probes.
\end{abstract}

Supplementary material available online at http://jeb.biologists.org/cgi/content/full/215/9/1523/DC1

Key words: insect, vision, blowfly, gaze, head movement, body movement, compensation, stabilization, walking, structured substrate.

\section{INTRODUCTION}

Locomotion in a three-dimensional (3-D) world causes complex retinal image shifts. These may be strongly affected by involuntary eye displacements induced by the properties of the locomotor apparatus. For instance, leg and body kinematics of humans even during walking on flat ground would cause periodic vertical head and eye displacements and, accordingly, vertical retinal image shifts, if the head and eyes were tightly coupled to the body (Grossman et al., 1988; Pozzo et al., 1990; Hirasaki et al., 1999). Such vertical image shifts would complicate object fixation and extraction of pattern details of the surrounding environment. Another example is the wing stroke of flying animals, which also produces involuntary head and eye displacements (van Hateren and Schilstra, 1999a). Moreover, for kinematic reasons, many fastflying animals have to perform banked turns by rolling their body to the side while rotating around their transverse body axis (pitching) towards the desired direction. Flying blowflies may reach roll angles of $90 \mathrm{deg}$ during large banked turns (Wagner, 1986; van Hateren and Schilstra, 1999a; van Hateren and Schilstra, 1999b; Geurten et al., 2010). If their head and eyes were tightly coupled to their body, the orientation of their visual system would rotate in space as well, complicating the processing of information about the external world.

To reduce these negative consequences of unintended locomotioninduced eye displacements, animals perform compensatory head and eye movements. These are mostly reflexive and countervail changes of the body position [head bobbing in birds (Necker, 2007)] and orientation in space. Thereby they stabilize gaze to enable, for instance, precise object fixation during locomotion (Dunlap and
Mowrer, 1930; Land, 1973; Pozzo et al., 1990; Miles, 1997; van Hateren and Schilstra, 1999b).

Compensatory head and eye movements appear to be multimodally controlled. The visual system is thought to register unintended whole-field image shifts and initiates movements to reduce the retinal image slip (Wallmann and Letelier, 1993; Hengstenberg, 1993; Boeddeker and Hemmi, 2010). Concurrently, diverse mechanosensory systems sense self-movements - changes in body attitude and head orientation - to induce corrective head movements, usually in a different dynamic range than the visual system [primates (Waespe and Henn, 1987); birds (Zeigler and Bischof, 1993); insects (Hengstenberg, 1993)].

Walking animals have a special problem in keeping their gaze stable, because under natural conditions they frequently have to walk on structured and bumpy substrates. Kinematics of the walking apparatus and direct contact with the ground and its asperities may cause strong unintended body movements, which would degrade the stability of gaze. As insects cannot move their compound eyes independently of their head, they perform exclusively compensatory head movements. Tethered and freely walking blowflies were shown to perform compensatory head movements on flat ground to stabilize gaze (Horn and Lang, 1978; Hengstenberg, 1986; Blaj and van Hateren, 2004). However, it is still unclear to what extent walking insects are able to hold their gaze stable when walking on structured ground. Furthermore, because of the complex multimodal control of head movements, it is unknown to what extent the visual system is involved in controlling these movements.

To tackle these knowledge gaps of the control and operating range of gaze stabilization in insects, we analyzed compensatory head 
movements of freely walking blowflies. The blowfly Calliphora vicina Robineau-Desvoidy 1830 has served for many years as a model system for analyzing compensatory head movements and visual information processing (for reviews, see Land, 1999; Egelhaaf, 2006; Borst et al., 2010). Walking is an essential behavioral mode of blowflies, because they perform near-field food search behaviors as well as oviposition on ground (Dethier, 1976). To analyze compensatory head movements, we recorded head and body movements during straight walks on differently structured substrates ('walking bars'). The surface of these walking bars varied from flat to coarsely structured, with bumps of up to nearly the size of the animal. To assess the role of the visual system for the control of compensatory head movements, we compared rotational head stability of flies walking in the dark and under bright lighting conditions.

\section{MATERIALS AND METHODS Flies and preparation}

We used female blowflies C. vicina from stocks reared in our laboratory. The flies were caught out of the stock cage 1 day post eclosion. To prevent them from flying, we briefly anaesthetized them with $\mathrm{CO}_{2}$ and placed a drop of beeswax on each wing base. We painted three marker points on their head and six points on their thorax using white, nontoxic acrylic paint (Hooby Line, C. Kreul, Hallerndorf, Germany), to allow automatic tracking of head as well as body position and orientation (Fig. 1A). Prior to final marker placement, we cut the longest hairs at the intended locations to optimize marker bonding to the cuticle. Before the first experiment, flies had at least $24 \mathrm{~h}$ in isolation to adapt to the wing fixation and marker placements.

\section{Experimental setup \\ Filming setup}

We recorded walking blowflies at 500 frames s$^{-1}$ with two orthogonally arranged and synchronized infrared (IR)-sensitive highspeed cameras (CR 600, Optronis GmbH, Kehl, Germany), both equipped with DG MACRO 24-70 mm lenses (SIGMA GmbH, Roedermark, Germany). One camera was positioned $50 \mathrm{~cm}$ above the walking bar (top view). The other camera was positioned $38 \mathrm{~cm}$ in front of the bar behind a hole $(4.2 \mathrm{~cm}$ diameter) in a white screen, so that the recorded flies walked directly towards it (frontal view). The walking bar was placed on a custom-made light table, which illuminated the scenery from below. In addition, we placed two LED panels (Marathon multiLED, VIDEAL, Niederoenz, Germany) $60 \mathrm{~cm}$ above the walking bar (Fig. 2A). A mirror $(10 \times 5 \mathrm{~cm})$ was fixed to the right side of the walking bar at an angle of $45 \mathrm{deg}$, which provided a side view of the walking blowflies from the top camera. To avoid a potential bias on head or body orientation caused by the unilaterally positioned mirror, we placed a second, identical mirror along the left side of the walking bar.

For recordings in the dark, we replaced the multiLED light sources with two custom-made IR LED panels, each consisting of 70 single IR LEDs (GaAIAs Double Hetero, Vishay Electronic GmbH, Selb, Germany; peak emission at $\lambda=890 \mathrm{~nm}$ ). The IR LED panels emitted long-wave light far above the perceivable wavelengths of the blowfly's photoreceptors [R1-R6 sensitive to light up to $600 \mathrm{~nm}$; $\mathrm{R} 7$ and R8 sensitive to light up to 500 and $600 \mathrm{~nm}$, respectively (Hardie, 1979)], but within the sensitivity range of our IR-sensitive high-speed cameras (sensitive up to $1000 \mathrm{~nm}$ ). We placed the IR LED panels approximately $17 \mathrm{~cm}$ to the left and right above the walking bar. All potential additional light sources (e.g. the indication LEDs of the cameras), which might be used by the flies for

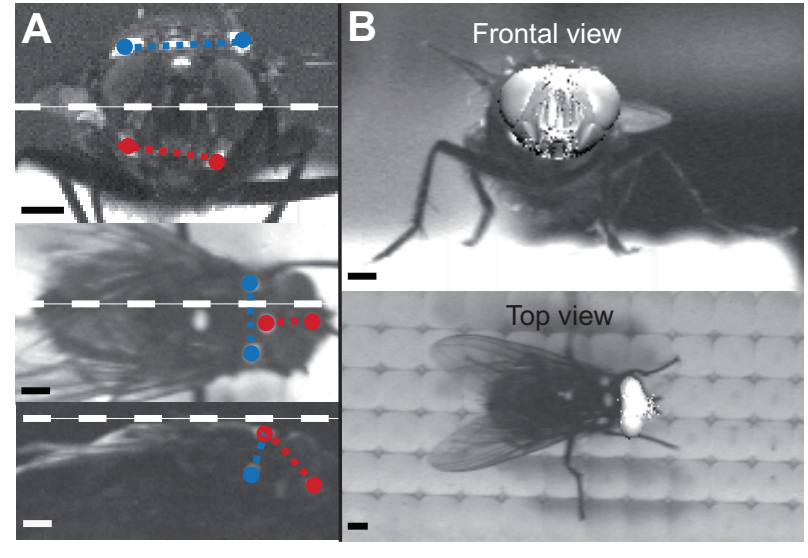

Fig. 1. (A) Marker positions and angle calculation. Marker positions on the fly's head and body are indicated by red and blue dots, respectively. The vectors necessary to calculate the orientation angles $\Phi$ of the head and body are illustrated by the dashed lines. The red lines indicate the calculated orientation vector $\mathbf{v}_{1}$ of the head, the blue lines the orientation vector $\mathbf{v}_{1}$ of the body, and the white lines the respective reference vector $\mathbf{v}_{2}$ (Eqn 1). The frontal view (top) was used to calculate roll orientations, the top view (center) to calculate the yaw orientations and the side view (bottom) to calculate the pitch orientations of the head and the body. (B) Illustration of the three-dimensional (3-D) method, in which the frontal and top view video images were overlaid with a transparent 3-D model of the blowfly's head. The model was rotated until its orientation matched the head orientation on the images beneath. Note that for illustrative reasons the head model is presented here not transparently. Scale bars, $1 \mathrm{~mm}$.

orientation, were covered with black insulating tape. Only an indirect red light source (PF 712E, Philips Deutschland GmbH, Hamburg, Germany) was used to enable the experimenter to handle the blowflies in the experimental setup. To assess what perceivable light intensities were present in the walking setup, we measured the luminance on the walking bar with a luminance meter (Konica Minolta Sensing LS-100, Osaka, Japan). Note that although the luminance meter was adapted to the sensitivity of the human eye, it provides a rough estimate of the light perceived by the fly because of the similar spectral sensitivities of human vision and fly photoreceptors (Riggs et al., 1949; Hardie, 1979). In the dark condition, the luminance amounted to $0.01 \mathrm{~cd} \mathrm{~m}^{-2}$, i.e. more than five orders of magnitude below the luminance of the bright condition $\left(4820 \mathrm{~cd} \mathrm{~m}^{-2}\right)$.

\section{Structured walking bars}

The walking bars were made of gypsum, which was poured into milled aluminum molds under vacuum to prevent air pockets. We designed four mold forms with a custom script written in MATLAB (The MathWorks, Natick, MA, USA) that was capable of producing computer-aided design files. All bars had a length of $12 \mathrm{~cm}$ and were $1.6 \mathrm{~cm}$ wide (Fig. 2B). The walking bars were narrow enough to force the flies to walk straight, but wide enough to prevent them from simultaneously grabbing the left and right edges of the bar. One bar had a flat surface and served as the control condition to estimate head and body movements induced exclusively by the walking apparatus. The other three walking bars had surfaces consisting of regularly placed convex hemispheres ('bumps'). The diameters of the bumps defined the substrates' structure. We term the flat surface ' $0 \mathrm{~mm}$ substrate' and the others ' $3 \mathrm{~mm}$ substrate', ' $6 \mathrm{~mm}$ substrate' and ' $8 \mathrm{~mm}$ substrate' (Fig. 2B, left to right). Each walking bar was mounted on a $10 \mathrm{~cm}$ high stand (Fig. 2A). 

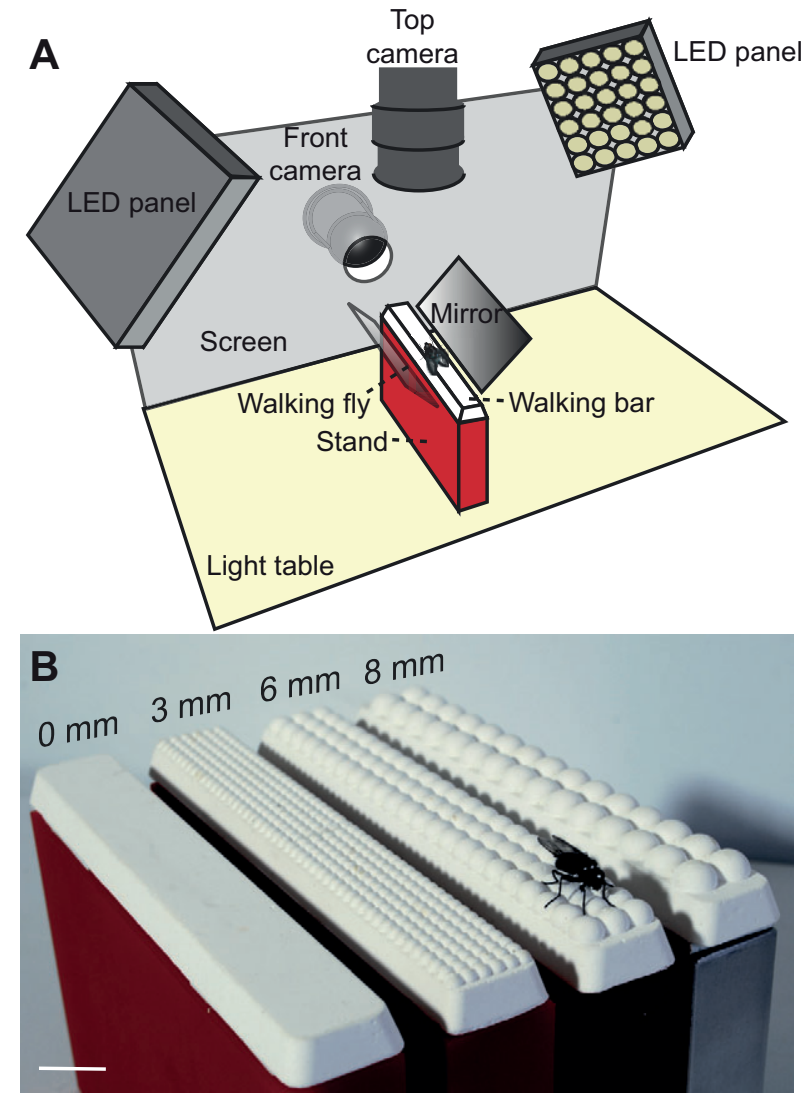

Fig. 2. (A) Scheme of the experimental setup (not drawn to scale). Blowflies were recorded using two synchronized high-speed cameras ( 500 frames s$^{-1}$ ) during walks across the walking bar. A mirror was placed parallel to the substrate to obtain a side view of the walking fly.

(B) Structured walking bars. The four different substrate types consisted of regularly placed hemispheres (bumps) ranging in diameter from 0 to $8 \mathrm{~mm}$. The bars had a length of $12 \mathrm{~cm}$, a width of $1.6 \mathrm{~cm}$ and were mounted on $10 \mathrm{~cm}$ high stands. Scale bar, $1 \mathrm{~cm}$.

\section{Experimental procedure}

Flies were transferred from the adapting cage using transparent plastic vessels $(2 \times 6 \mathrm{~cm}$, diameter $\times$ length $)$ and released at the far end (as seen from the frontal camera) of the walking bar. Eighty percent of the released flies started walking right away. After the tested fly reached the opposite end of the walking bar we caught it with the vessel, examined the recorded walk and saved it on hard disk in case the walk satisfied certain criteria: (1) the fly had to walk continuously without interruption while traversing the depth of field segment of the frontal camera; (2) the fly had to walk on top of the substrate, so all six legs were placed on top of the walking bar and not on the sides at all times; (3) the fly performed at least three complete stride cycles of its tripod gait (Strauss and Heisenberg, 1990) in the depth of field area of the frontal camera, which covered approximately $3.5-4 \mathrm{~cm}$; and (4) head and body markers could be seen during the whole walk. We recorded three walks of 10 flies on each of the four substrates. To obtain more reliable individual data for each substrate condition, we averaged the respective data from the three walks for each individual fly. The substrate types were employed in pseudorandom order.

The experiments under IR illumination were conducted in the same way as described above, except that we accepted also walks with only two complete tripod stride cycles. This change was necessary to obtain a sufficient amount of data, because in darkness, flies were less motivated to start walking and, when walking, the distances covered tended to be shorter.

\section{Video analysis}

The recorded high-speed videos were analyzed frame by frame using the open source software ivTrace (http://opensource.citec.de/projects/ivtools) developed in the Neurobiology Department at Bielefeld University, which automatically tracked the centroid of the white marker point area on the blowfly's head and body, respectively. We used custom-written MATLAB scripts to calculate head and body positions and orientations for all video frames ('2D method'). We estimated the accuracy of the calculated orientation angles by shifting the marker positions by 1 pixel in different directions. In the worst case the orientation error was approximately $2 \mathrm{deg}$. Because the tracking software detects the center of mass of the marker points, tracking errors smaller than 1 pixel (approximately $1 \mathrm{deg}$ ) may be obtained. Fig. 1A illustrates the marker points taken for the computation of head and body yaw, pitch and roll angles, respectively. The angle $\Phi$ of the different rotation axes of the blowfly's head and of the body is given by the dot product of vector $\mathbf{v}_{1}$, i.e. the line between two marker points, and a reference vector $\mathbf{v}_{2}$, i.e. the default null orientation of the respective rotation axis:

$$
\Phi=\cos ^{-1}\left(\frac{\mathbf{v}_{1} \mathbf{v}_{2}}{\left|\mathbf{v}_{1} \| \mathbf{v}_{2}\right|}\right) \frac{180}{\pi} .
$$

To reduce jitter in the pixel coordinate data of the maker points, we filtered them (Gaussian-like filter: window size $6 \mathrm{~ms}$ corresponding to three data points, sigma $=2 \mathrm{~ms}$ ). The positions of the manually applied markers differed minimally between animals. Hence, level orientations of the head and body with respect to pitch, yaw and roll calculated for different individuals were afflicted with an offset and could only be pooled after a correction. We corrected for these systematic differences for each individual by adding to each calculated head angle the difference between calculated orientation and actual head and body orientation in the video image. Therefore, we selected frames in which the head and body were held in normal (i.e. not rolled, pitched or yawed) orientation. The correction angles ranged from -2 to $3 \mathrm{deg}$. These differences affect only the absolute orientations, not their relative changes. The 3-D trajectory of the fly's head was determined by stereo triangulation of the positions of the head marker located directly anterior to the ocelli in the corresponding 2-D images from both cameras (Fig. 1A). To define the 3-D space filmed by both cameras, the cameras had to be calibrated [using J. Y. Bouguet's MATLAB camera calibration toolbox; www.vision.caltech.edu/ bouguetj/calib_doc/ (Bouguet and Perona, 1998)]. By reconstruction of points in space of a known distance, we estimated the reconstruction error as, on average, $0.03 \mathrm{~mm}$.

Head rotations around more than one axis led the head to turn out of the projection plane of the frontal and/or top camera. Consequently, distortions could have emerged and affected the calculated head orientation. To assess this potential error of the 2D method, we introduced a partly transparent 3-D head model into ivTrace, which was positioned virtually over the frontal and top image. We manually adjusted the model's position and orientation framewise, so that it matched the position and orientation of the fly's head in the images (Fig. 1B). After this time-consuming routine, the orientation data could be extracted from the 3-D model trace. We call this technique the ' 3 -D method'. To estimate the error introduced by this procedure, the experimenter repeated the 3-D method for a given segment (30 frames) of the fly's trajectory on five consecutive days. The mean standard deviation between the 3$\mathrm{D}$ adjustments was $1.2 \mathrm{deg}$. 
Because the 3-D method is assumed to yield rotation angles closer to the real ones at the expense of analysis time, we determined its benefit against the rotation angles determined with 2-D method. We calculated the mean difference angle $\Delta \Phi$ and the correlation coefficient $r$ between both time-dependent rotation data sets. The results were as follows: pitch, $\Delta \Phi=0.26 \mathrm{deg}, r=0.94$; roll, $\Delta \Phi=0.74 \mathrm{deg}, r=0.91$; and yaw, $\Delta \Phi=2.58 \mathrm{deg}, r=0.75$. The relatively large $\Delta \Phi$ value and low correlation of the yaw data indicate a discrepancy for head yaw data obtained with the 2-D and 3-D methods. Therefore, we used the automatic 2-D method to obtain positional as well as pitch and roll data of the flies' head and thorax. However, head yaw angles were determined with the more timeconsuming 3-D method. Jitter in the head yaw data was reduced by filtering with a Butterworth filter (second degree; relative cut-off frequency $0.1 \mathrm{~Hz}$ ). We verified that this cut-off frequency had no functionally distorting effect on the yaw data. The stride cycles were determined manually by noting the touchdown time point of the left foreleg (McNeill, 2003), and were used to calculate the average stride frequency and the stride-related head and body rotations.

\section{Statistical tests}

Under bright light conditions, each tested fly walked three times on each of the four substrates. To compare the paired data obtained on the different substrates, we used multivariate ANOVA (MANOVA) for repeated measurements. Data were tested for normality with the Lilliefors test. Single variables, as roll or vertical translational fluctuations, were tested with an ANOVA for repeated measurements. All data sets have been tested with the Box test or the Mauchly test for homogeneity of covariance or variance, respectively. For heterogeneous covariances or variances, we give the Greenhouse-Geisser corrected MANOVA or ANOVA results. The Bonferroni's correction for multiple comparisons (BC) was applied as post hoc test. Changes of body posture between the light conditions were analyzed with a $t$-test. The error values are given as standard deviation (s.d.). Statistical tests were performed with IBM SPSS Statistics (IBM Deutschland GmbH, Ehningen, Germany) and the statistics toolbox of MATLAB.

\section{RESULTS}

\section{Blowfly compensatory head and body movements when walking on differently structured substrates}

We recorded 10 flies ( $N=$ individual flies) walking three times ( $n=$ total recorded walks) on the four different substrates $(0,3,6$ and $8 \mathrm{~mm}$ bumps). Within the field of view of the cameras, flies walked on average a distance of $3.64 \pm 0.12 \mathrm{~cm}$, which corresponds to approximately four times their body length (approximately $9 \mathrm{~mm}$ ).

The time course of body and head locomotion parameters is depicted in Fig. 3 for a single run on the $0 \mathrm{~mm}$ substrate. The fly had an average walking speed of $17.5 \mathrm{~cm} \mathrm{~s}^{-1}$ (Fig. 3B) and walked a distance of $4.6 \mathrm{~cm}$. The head and body rotated slightly around the three rotation axes. Pitch and roll rotations of the head were smaller than the body rotations (Fig. 3A), revealing that the head was held more stable than the body (supplementary material Movie 1). Head and body rotations around the yaw axis (Fig. 3A) drifted during this walk to slightly negative values, indicating slightly rightward turning of the head and body. The fluctuations (s.d.) of body roll orientation were, at $5.8 \mathrm{deg}$, more than three times larger than the corresponding head roll fluctuations of $1.7 \mathrm{deg}$ (Fig. 3A).

Besides rotations, we also analyzed head translations (Fig. 3B). Similar to head orientation and rotation velocities, head position and forward velocity fluctuate during walking. The head of the
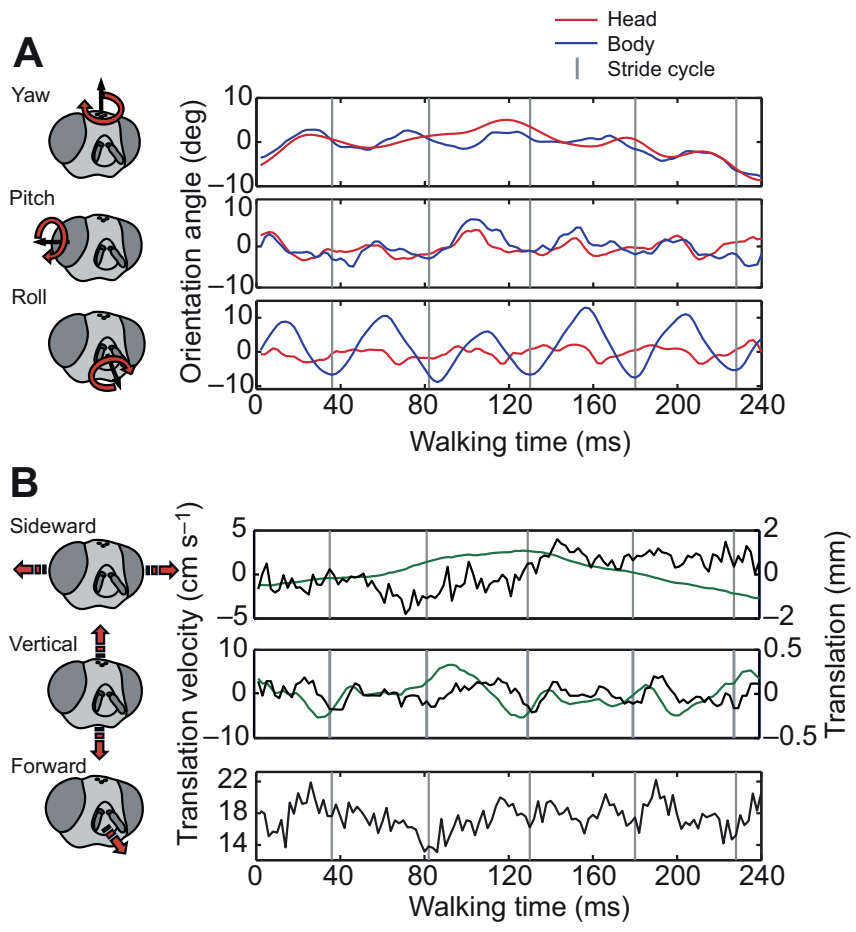

Fig. 3. Individual head and body rotation and translation data for a walk on even substrate. Note that for illustrative purposes a walk with large fluctuations is presented. (A) Head and body rotations around the yaw, pitch and roll axes. Positive values indicate leftward turns for yaw, upward turns for pitch and rightward turns for roll rotations whereas negative values indicate rotations in the opposite directions. (B) Calculated translational head velocities (black line) as well as sideward and vertical head translations (green line). Positive translations indicate leftward movements for sideward and upward movements for vertical translations. Vertical gray lines depict the left foreleg touchdown time as an indicator of the start of a new stride cycle. Data were obtained at 500 frames s$^{-1}$.

walking fly experienced a maximal sideward shift of $2.2 \mathrm{~mm}$ at velocities $\leq 4.5 \mathrm{~cm} \mathrm{~s}^{-1}$ and a maximal vertical shift of $0.6 \mathrm{~mm}$ with velocities of up to $5.1 \mathrm{~cm} \mathrm{~s}^{-1}$. During this walk, forward velocities never decreased below $13 \mathrm{~cm} \mathrm{~s}^{-1}$ and reached peak values exceeding $22 \mathrm{~cm} \mathrm{~s}^{-1}$ (Fig. 3B).

\section{Relationship between stride cycle and head and body rotations}

Rotational and translational head and body fluctuations contain a strong periodic component that may be caused by a coupling between head and body rotations and the stride cycle. The length of a full stride cycle (i.e. all six legs performed a step in a tripod gait) was determined from the time when the left foreleg touches the ground. The duration of a stride cycle as averaged across all flies walking on flat ground was $67.4 \pm 0.5 \mathrm{~ms}$. To analyze the coupling between stride cycle and rotational fluctuations, we averaged the head and body rotation data within a time window of $34 \mathrm{~ms}$ (half the mean stride cycle length) before and after a completed stride cycle. The respective mean rotation values were subtracted to allow averaging over different walks. In this way, we averaged for each walk across three to seven stride cycles (mean completed stride cycles: $4.8 \pm 0.2$ ) and then across the three walks performed by each fly per substrate. Note that non-periodic rotational head and body fluctuations are averaged out by this method. To determine how the different substrates influence the stride-induced 
head and body rotations, we averaged the data of all walking flies separately for the different substrates (Fig. 4).

In general, the influence of the stride cycle on rotations was equally strong for head and body yaw, whereas roll and pitch rotations of the body were affected more strongly than the corresponding head rotations. On the $0 \mathrm{~mm}$ substrate, the body roll rotations coupled to the stride cycle had amplitudes of approximately $8 \mathrm{deg}$; the corresponding head roll rotations had amplitudes of less than half that value, approximately $3 \mathrm{deg}$ (Fig. 4).

The amplitudes of rotations coupled to the stride cycle became smaller with increased substrate granularity of the ground (Fig. 4). For instance, pitch fluctuations of head and body had amplitudes of $2.5 \pm 0.9$ and $4.4 \pm 1.2 \mathrm{deg}$, respectively, on $0 \mathrm{~mm}$ substrate, but $2 \pm 0.9$ and $3 \pm 1.2 \mathrm{deg}$ on $8 \mathrm{~mm}$ substrate. At first sight, this result might seem counterintuitive, because head and body rotations might be assumed to increase with increasing substrate granularity. However, note that the fluctuations coupled to the stride cycle do not represent the total head rotations (non-periodic rotational head and body fluctuations are averaged out). With increasing substrate granularity, the total rotational fluctuations became slightly larger (see next section). In other words, the effect of the substrate granularity on head and body rotations increased, whereas the effect of the walking apparatus on head and body rotations decreased on more coarsely structured substrates.

\section{Structure dependence of rotation fluctuations}

In general, head and body stabilization were diversely affected during walking on differently structured substrates (MANOVA, $\left.F_{1,9}=26.5, P=0.001\right)$. Although head fluctuations were approximately $0.8 \mathrm{deg}$ smaller than body fluctuations around the pitch and approximately $1.8 \mathrm{deg}$ around the roll axis, indicating compensatory head movements (Fig. 5), head yaw fluctuations largely overlap with those of the body (Fig. 3A, Fig. 5) and were, on average, $0.31 \mathrm{deg}$ larger on all substrate types.

Head and body fluctuations significantly increased with substrate granularity (MANOVA, $F_{3,27}=12.3, P<0.001$; Fig. 5). Hence, the tested surface structures degraded the rotational stabilization of the fly's body and that of the fly's head, which was especially prominent for rotations around the pitch axis (Fig.5A). Pitch fluctuations increased monotonically with substrate granularity from 2.4 to $4.2 \mathrm{deg}$ for the head and from 3.2 to $4.7 \mathrm{deg}$ for the body. In contrast, roll fluctuations stayed more or less constant on the 0,3 and $6 \mathrm{~mm}$ substrates and only increased noticeably on the $8 \mathrm{~mm}$ substrate (ANOVA, body, $F_{3,27}=3.04, P=0.087$; head, $F_{3,27}=3.22, P=0.089$; Fig. 5A). Accordingly, compensation around the roll axis seemed to be the most robust during walking on structured substrates.

To assess rotational head and body velocities during walking, we calculated the time derivative of all recorded head and body orientation data. We took the absolute values to calculate the mean rotational velocities. Head rotations were significantly slower than body rotations around all rotation axes and on all substrates (MANOVA, $F_{1,27}=57.2, P<0.001$; Fig. 5B). Pitch and roll head rotations were approximately $25 \%$ slower than those of the body, whereas head yaw turns were approximately $11 \%$ slower. Moreover, yaw rotations were on average the slowest turns whereas roll turns were the fastest, with velocities of up to $>1000 \mathrm{deg} \mathrm{s}^{-1}$ in exceptional cases. These results are confirmed by the probability densities of the rotational velocities (Fig. 5C). Low rotational head velocities (up to $200 \mathrm{deg} \mathrm{s}^{-1}$ ) occurred more often than low rotational body velocities. The effect of substrate granularity on rotational velocities was significant (MANOVA, $F_{3,81}=5.05, P<0.001$ ) but less systematic than on the rotational fluctuations.

\section{Structure dependence of translation fluctuations}

To assess how well the flies' head is stabilized in space during walks on structured ground, we determined head translations from individual head trajectories (Fig. 6). The translational forward head velocity or walking speed was significantly affected by the substrate granularity (ANOVA, $F_{3,27}=10.66, P<0.001$; Fig. 6B). Flies walked slightly faster on the $3 \mathrm{~mm}(\mathrm{BC}, P=0.044)$ and $6 \mathrm{~mm}(\mathrm{BC}, P=0.12)$ substrates than on flat ground. In fact, walking speed was fastest on the $3 \mathrm{~mm}$ substrate and slowest on the $8 \mathrm{~mm}$ substrate. The fluctuations of head sideward translations were similar for all substrates and ranged from 0.82 to $1.1 \mathrm{~mm}$ (ANOVA, $F_{3,27}=2.38$, $P=0.09$; Fig. 6A). The head of a blowfly has a typical width of approximately $3.5 \mathrm{~mm}$. Thus, the recorded head sideward translations did not exceed half head width. Vertical head translations (Fig. 6A) were significantly affected by substrate structure (ANOVA, $\left.F_{3,27}=18.58, P<0.001\right)$ and increased monotonically with substrate granularity. At $0.44 \pm 0.12 \mathrm{~mm}$, they were more than twice as large on the $8 \mathrm{~mm}$ substrate compared with on flat ground $(0.2 \pm 0.03 \mathrm{~mm})$. Nevertheless, in general, vertical translations were small during

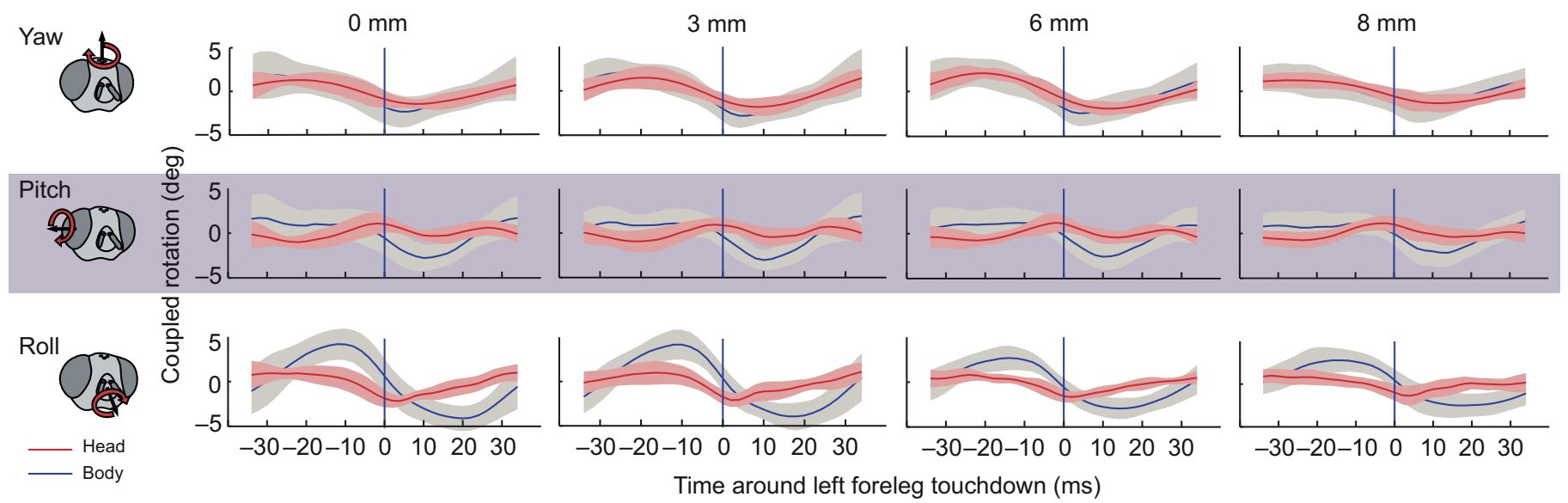

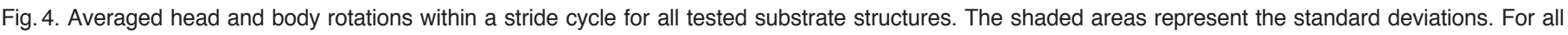
walks, the rotation data $34 \mathrm{~ms}$ (half the mean stride cycle length) before and after a completed stride cycle (vertical blue line at $0 \mathrm{~ms}$ ) were averaged. The individual mean orientation angles were subtracted to allow averaging over different walks ( $N=10$ flies, $n=118$ recorded walks). Positive values indicate leftward turns for yaw, upward turns for pitch and rightward turns for roll rotations while negative values indicate rotations in the opposite directions, respectively. 

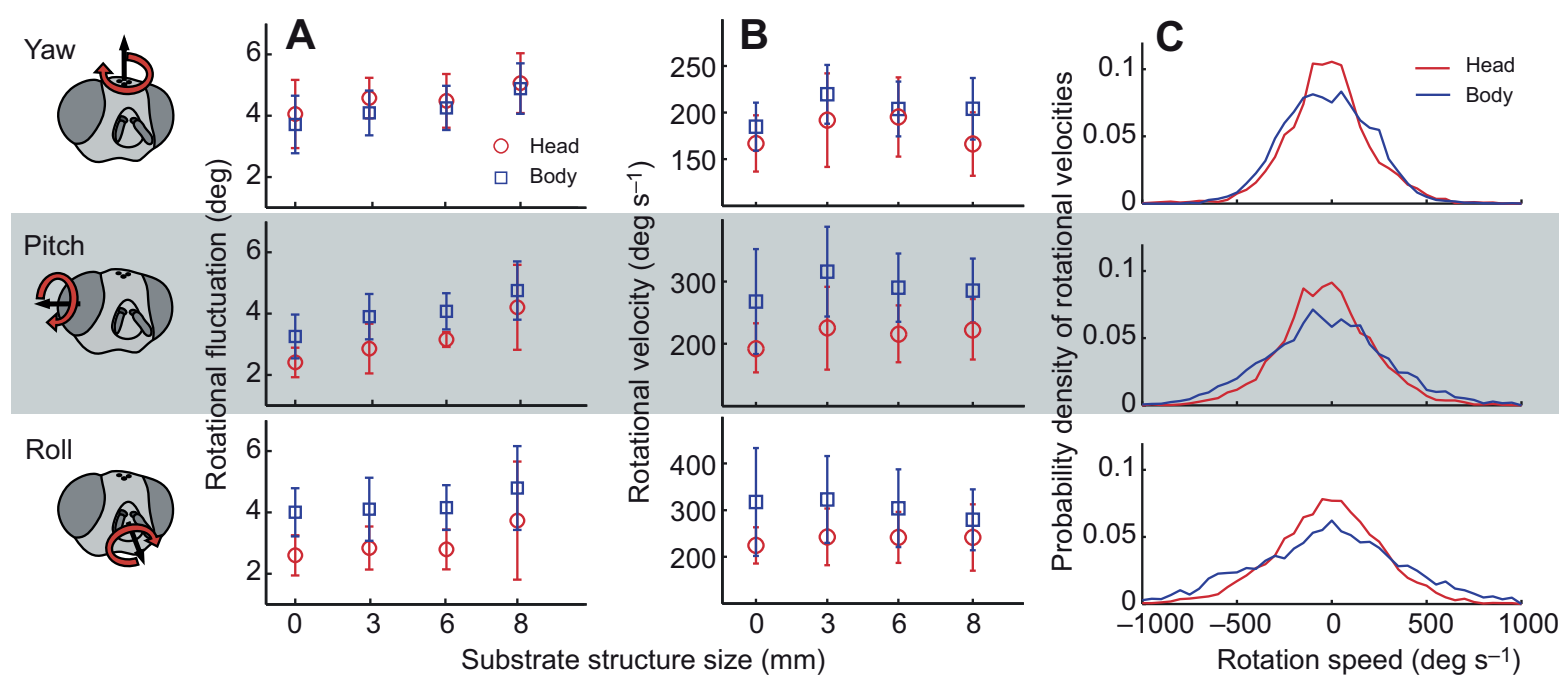

Fig. 5. (A) Mean ( \pm s.d.) rotational head and body fluctuations of blowflies walking on differently structured substrates. Rotational head and body fluctuations were significantly affected by substrate structure size (MANOVA, $F_{3,27}=12.3, P<0.001$ ). Moreover, head fluctuations differed significantly from body fluctuations (MANOVA, $F_{1,9}=26.5, P=0.001$ ). (B) Mean ( \pm S.d.) head and body rotation velocity. Ground structure had a significant effect on the rotational velocity (MANOVA, $F_{3,81}=5.05, P<0.001$ ). Head rotations were significantly slower than body rotations (MANOVA, $\left.F_{1,27}=57.2, P<0.001\right)(N=10$ flies, $n=118$ recorded walks). (C) Probability density distribution of head and body rotation velocities. Data are presented for the $0 \mathrm{~mm}$ condition. Note that probability densities did not differ considerably between the substrate types. Data are binned in classes of $50 \mathrm{deg} \mathrm{s}^{-1}$.

walking, even on the $8 \mathrm{~mm}$ substrate, given the fact that the corresponding bumps had nearly the size of the fly's body.

Translational mean velocities were calculated as the derivative of the head position over time. We found a weak effect of substrate granularity on sideward head velocities (ANOVA, $F_{3,27}=3.94$, $P=0.019$; Fig. 6B). Vertical head translation velocities also increased significantly with substrate granularity (ANOVA, $F_{3,27}=5.17$, $P=0.006$; Fig. 6B).

\section{Benchmark of compensation}

To assess to what extent head and body are stabilized by compensatory mechanisms, we need to know how much they would fluctuate without such compensatory mechanisms. Because uncompensated fluctuations cannot be determined directly, we roughly estimated their potential size by comparing the granularity of the surface structure (i.e. height of substrate bumps) and the observed fluctuations in vertical head translations. We assumed that the vertical translations of the body are completely transferred to the head because head movements mediated by the fly's neck motor system are mainly rotational and not translational (Sandeman, 1980a). Furthermore, we subtracted the vertical translational fluctuations recorded on the flat surface from the fluctuations determined for the structured substrates to assess the vertical translation values due to substrate granularity. The calculated vertical head translations induced by the substrate were $0.07 \mathrm{~mm}$ for the $3 \mathrm{~mm}$ substrate, $0.15 \mathrm{~mm}$ for the $6 \mathrm{~mm}$ substrate and $0.23 \mathrm{~mm}$ for the $8 \mathrm{~mm}$ substrate. These substrate-induced fluctuations amount, on average, to only $5.2 \%$ of the respective height of the hemispheres, which were used to characterize the different walking substrates. In other words, the walking apparatus of the tested blowflies compensates for most of the granularity of the walking surface. This good compensation is likely to be both, controlled via sensory systems and a consequence of leg properties and step positions on the substrates. More elaborate analyses would require sophisticated models of the blowflies' pedal system and of the structured substrates. The generation of such models is far beyond the scope of the present study.

\section{Relationship between stride frequency and walking speed}

The mean stride cycle frequency over all conditions was $14.83 \pm 2.24 \mathrm{~Hz}$. We found a strong positive correlation between walking speed and stride frequency for all substrate types $\left(r_{0 \mathrm{~mm}}=0.89\right.$,
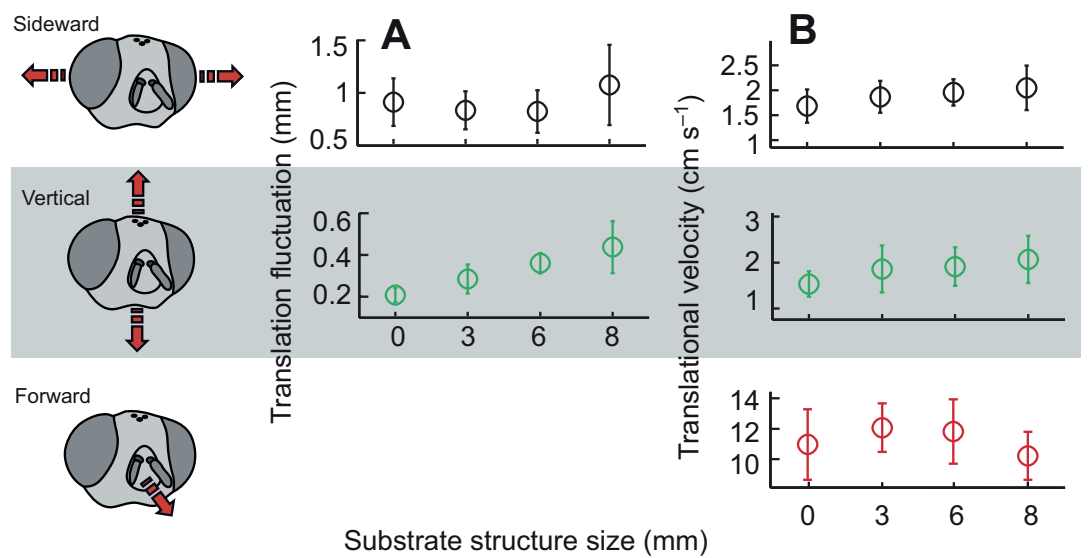

Fig. 6. (A) Mean ( \pm s.d.) translational head fluctuations of blowflies walking over differently structured substrates. Translational sideward fluctuations were not affected by substrate structure size (ANOVA, $F_{3,27}=2.38, P=0.09$ ) whereas vertical fluctuations are strongly affected (ANOVA, $F_{3,27}=18.58, P<0.001$ ). (B) Mean ( \pm s.d.) head velocity data of blowflies walking on differently structured substrates. Sideward, vertical and forward velocities are significantly affected by the ground structure (ANOVA, sideward, $F_{3,27}=3.94, P=0.019$; vertical, $F_{3,27}=5.17, P=0.006$; forward, $F_{3,27}=10.66, P<0.001$ ) ( $N=10$ flies, $n=118$ recorded walks).

Substrate structure size $(\mathrm{mm})$ 


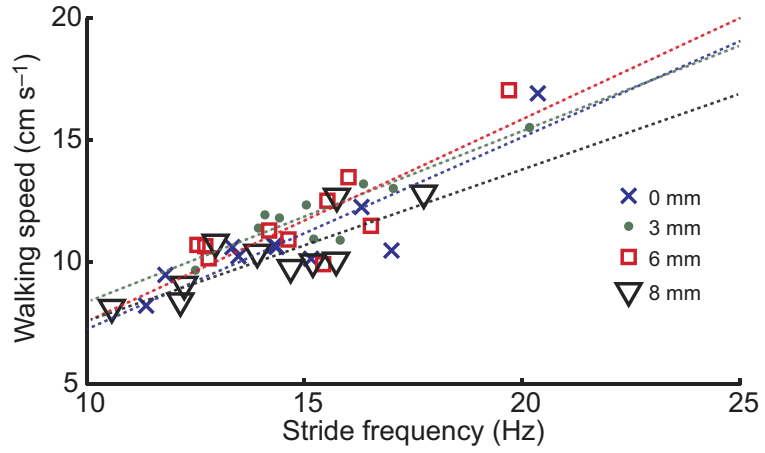

Fig. 7. Relationship between stride frequency and walking speed. Plotted are the mean stride frequencies and walking speeds of each tested fly $(N=10)$ on all substrate types $(10 \times 4=40)$. Linear regression fits are color coded (see key). Linear equation: walking speed $=m \times$ stride frequency $+b$ (linear slope: $m_{0 \mathrm{~mm}}=0.77, m_{3 \mathrm{~mm}}=0.68, m_{6 \mathrm{~mm}}=0.82$ and $m_{8 \mathrm{~mm}}=0.61 ; y$ intercept: $b_{0 \mathrm{~mm}}=-0.54, b_{3 \mathrm{~mm}}=1.56, b_{6 \mathrm{~mm}}=-0.49$ and $b_{8 \mathrm{~mm}}=1.69 ; R_{0 \mathrm{~mm}}^{2}=0.79$, $R_{3 \mathrm{~mm}}^{2}=0.8, R_{6 \mathrm{~mm}}^{2}=0.7$ and $\left.R_{8 \mathrm{~mm}}^{2}=0.68\right)$.

$r_{3 \mathrm{~mm}}=0.89, r_{6 \mathrm{~mm}}=0.84$ and $r_{8 \mathrm{~mm}}=0.82 ;$ Fig. 7 ). The average stride length is represented by the slopes of the respective regression fits (0 mm: $0.77 \mathrm{~cm} ; 3 \mathrm{~mm}: 0.68 \mathrm{~cm} ; 6 \mathrm{~mm}: 0.82 \mathrm{~cm} ; 8 \mathrm{~mm}: 0.61 \mathrm{~cm}$ ), which differed significantly for the tested substrate conditions (ANOVA, $F_{3,27}=4.91, P=0.008$; Fig. 7). Post hoc tests revealed that this significant variance was restricted to the 6 and $8 \mathrm{~mm}$ conditions (BC, $6 \mathrm{~mm} v s 8 \mathrm{~mm}, P=0.03$ ), indicating that, on average, the tested flies made longer strides on the $6 \mathrm{~mm}$ substrate than on the $8 \mathrm{~mm}$ substrate. However, there was no systematic change in average stride length, as the calculated values did not monotonically increase or decrease with changes of the substrate granularity.

\section{Influence of the visual system on the control of head stabilization when walking on differently structured substrates}

To estimate the influence of the visual system on the compensatory head movements during walking on structured substrates, we eliminated the visual input of the flies by testing them in the dark. The only light sources were IR LED panels, which emitted longwave light far above the perceivable wavelengths of the blowfly's photoreceptors (Hardie, 1979). Because under bright light conditions the most pronounced difference in rotational fluctuations had been found between walks on the 0 and $8 \mathrm{~mm}$ substrates, we recorded walks of three flies on only these substrates in the dark. In general, flies were less motivated to start walking in the dark than under bright conditions. In addition, on average, their walks were $1.7 \mathrm{~cm}$ shorter $(\mathrm{BC}, P<0.001)$ and less directional compared with bright light conditions.

Another obvious difference between walks in light and in the dark was an altered head and body posture. In the dark, blowflies shifted their body towards the mid and hind legs, pitched their body and head upwards and performed extensive movements with their forelegs without using them directly for walking (Fig. 8). On average, the body was pitched upwards by approximately $15.4 \mathrm{deg}$ $(P=0.003)$ and the head by approximately $10.7 \mathrm{deg}(P=0.011)$ during walks in the dark compared with walks under bright light conditions on flat substrate. As a consequence, the forelegs had virtually no load to carry but acted as tactile probes to detect obstacles and aided leg positioning. This behavior was only observed in the dark.

Because of the upward-pitched body and head of flies walking in the dark, their head covered the body markers for the frontal camera. Therefore, it was not possible to analyze body roll rotations for walks in the dark. The fluctuations of the head and the two remaining body rotations are depicted in Fig. 9A. For roll, pitch and yaw, the head fluctuations increased on the $8 \mathrm{~mm}$ substrate. The average rotational fluctuations were similar under both lighting conditions (MANOVA, $F_{1,31}=0.204, P=0.65$ ).

The altered walking style of blowflies in the dark was accompanied by a reduction in average walking speed compared with walks in bright light (ANOVA, $F_{1,11}=8.12, P=0.016$; Fig. 9B). Flies tested in the dark walked approximately $3.4 \mathrm{~cm} \mathrm{~s}^{-1}$ slower on the flat substrate than flies in light. On the $8 \mathrm{~mm}$ substrate, flies in the dark walked at speeds of $5.6 \mathrm{~cm} \mathrm{~s}^{-1}$, only half as fast as in bright light.

\section{DISCUSSION}

Walking animals have direct mechanical contact with their substrate. Asperities in the surface structure of the substrate as well as the physical properties of the animal's walking apparatus should directly affect its head and body orientation, unless the animal somehow compensates for the unintended changes. Compensatory head

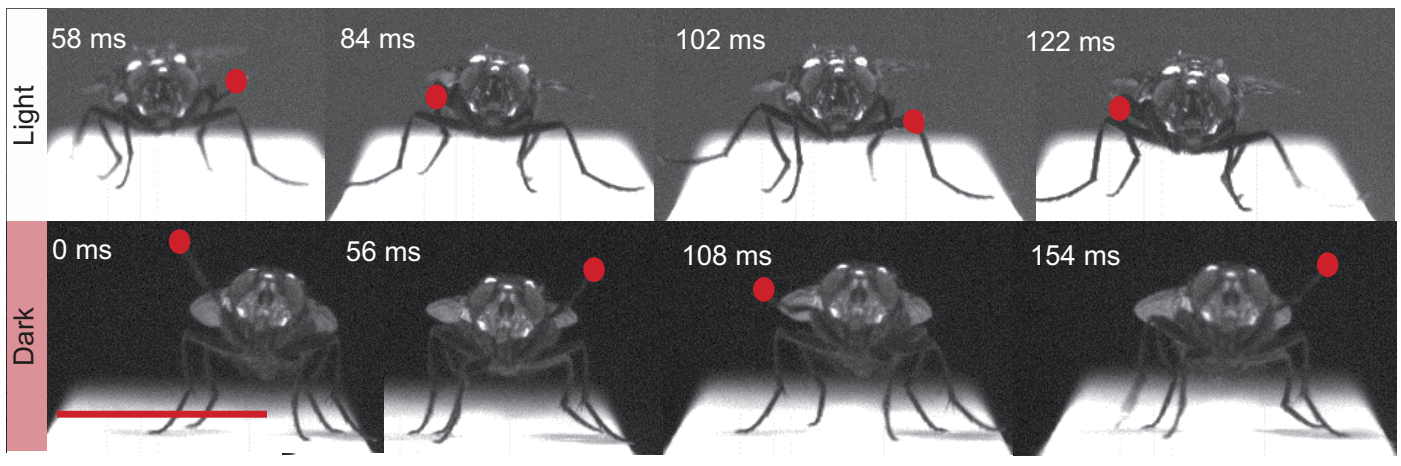

Fig. 8. Walking style differences under light and dark conditions. The upper row illustrates a walk under bright light. The row below illustrates a walk in the dark. Each row shows the highest foreleg swing (red dots) for four consecutive foreleg strides. Respective time in the video recording is shown in upper left

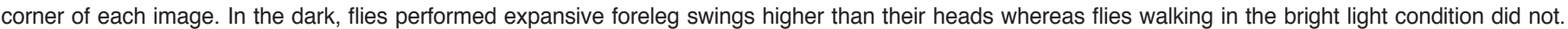
Note the higher upward pitched body $(14.9 \mathrm{deg})$ and head (11.3 deg) of the fly walking in the dark compared with the fly walking at bright light conditions. The fly walking under bright light conditions performed the four foreleg strides in $64 \mathrm{~ms}$ whereas the fly in the dark needed more than twice the time (154 ms). Scale bar, $1 \mathrm{~cm}$. We recorded different flies under light and dark conditions. 


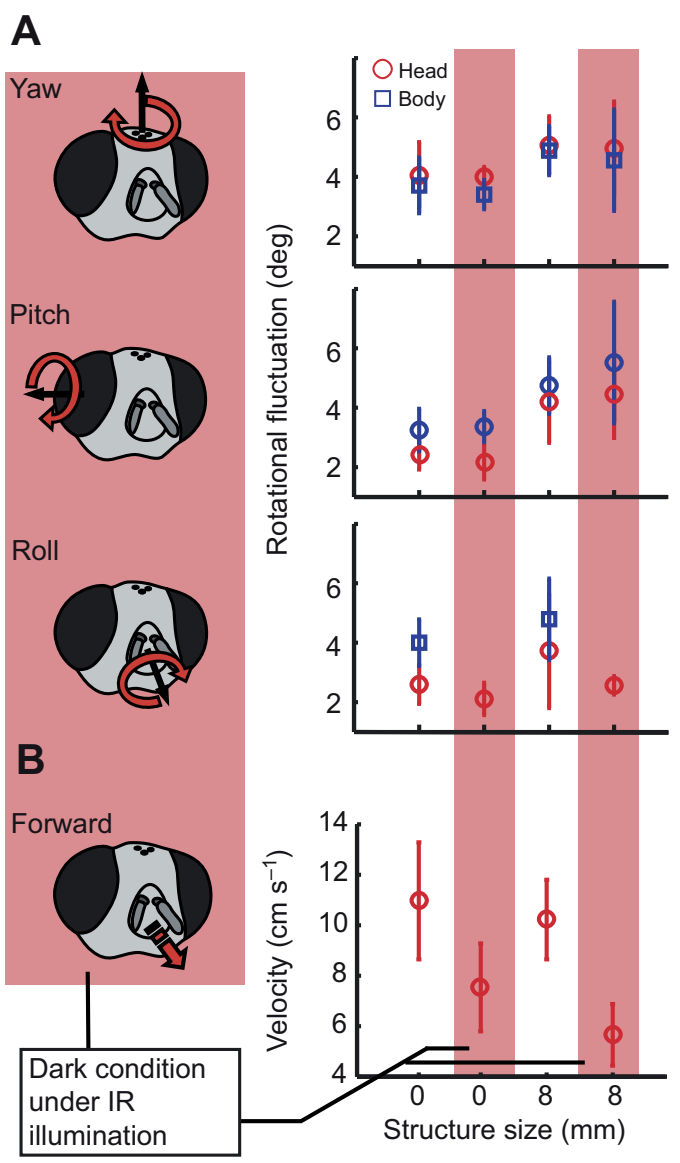

Fig. 9. (A) Rotation fluctuation comparison between flies walking on differently structured substrates under bright light conditions and in the dark without perceivable light (infrared illumination). Light condition had no significant influence on rotational fluctuation (two-way MANOVA,

$F_{1,31}=0.204, P=0.65$ ). (B) Walking speed on differently structured substrates under light and dark conditions. Forward velocity differed significantly between the conditions (two-way ANOVA, $F_{1,11}=8.12, P=0.016$ ) (light: $N=10$ flies, $n=58$ recorded walks; dark: $N=3$ flies, $n=16$ recorded walks).

movements might be important from a functional point of view as they may stabilize the orientation of gaze as a frame of reference for visual information processing. In this study, we addressed this issue for walking blowflies, which possess highly mobile heads and have been employed for many years successfully as a model system for analyzing visual information processing (Land, 1999; Egelhaaf, 2006; Borst et al., 2010) and compensatory head movements (Hengstenberg, 1993).

We found that body and head, and thereby gaze, orientation of freely walking blowflies are effectively stabilized, with residual rotational fluctuations ranging between 2 and $5 \mathrm{deg}$ around all axes. Surprisingly, even on coarsely structured ground with surface bumps as large as the animal, gaze stability was only marginally reduced for pitch and yaw and stayed constant for roll. For all tested surface conditions, head movements compensated for $25 \%$ of the rotational fluctuations of body pitch and $46 \%$ of the rotational fluctuations of body roll. Rotational head velocities were also reduced compared with body velocities by $26 \%$ for pitch, $22 \%$ for roll and $11 \%$ for yaw.

Head and body rotations of walking blowflies were found to be coupled, to some extent, to the stride cycle. This coupling was less prominent on strongly structured substrates. Stride cycle coupled rotations of the body (but not of the head) were recorded in an earlier study on freely walking blowflies (Horn and Mittag, 1980) and found to be in a range similar to those observed in the present study. Comparable observations have been made in humans, where vertical and horizontal head rotations that are coupled to stride frequency during walking are compensated, to some extent, to improve gaze stability (Grossman et al., 1988; Hirasaki et al., 1999).

What are the mechanisms underlying gaze stabilization on structured ground? The body fluctuations of blowflies originating from asperities of the walking substrate are compensated to a large extent by the pedal system. This conclusion is based on the finding that the translational and rotational body fluctuations increase only marginally as the bumps of the structured ground increase to nearly the animal's body size. On top of the already excellent body stabilization by the pedal system, the neck system of the blowfly performs additional compensatory head movements that stabilize the gaze even further (Figs 5, 6).

How is head and body stabilization in blowflies walking on structured substrates controlled? Because rotational head stability did not decrease in the dark (Fig. 9), we conclude that head and thus gaze stability in walking blowflies is not controlled visually but mainly by mechanosensory systems. This finding is surprising, as not only mechanosensory systems, but also the visual system, have previously been shown to induce compensatory head movements in tethered flight and walking (Hengstenberg, 1991; Hengstenberg, 1993). Based on older evidence of direct and indirect projections from wide-field motion-sensitive neurons of the third visual neuropile to neck motorneurons (Strausfeld and Seyan, 1982; Milde et al., 1987), more recent studies were able to show in which way the output from these neurons is used by the neck motor system and that this optomotor transformation depends on the behavioral state of the animal (Huston and Krapp, 2008; Schwyn et al., 2011; Rosner et al., 2010). Nonetheless, despite this evidence for compensatory optomotor head responses and their likely neural substrate, our data suggest that they do not play any obvious role in walking flies. Because substrate structure is not directly sensed by the visual system but by mechanosensory systems, it is apparently adequate to rely only on mechanosensory cues for controling compensatory body and head movements during walking on structured substrates.

The effective body stabilizing behavior by the pedal system is most likely controlled by mechanosensors in the legs' proprioceptive system. These have been shown in different insect species to be involved in the control of body orientation with respect to gravity (Horn, 1982) and in maintaining the distance between the body and the substrate (Kemmerling and Varju, 1981).

Head movements that compensate for unintended body movements that are induced by the ground structure and the walking apparatus are most probably controlled by the following systems: (1) the proprioceptive system in the forelegs and middle legs, which is also able to modulate compensatory head movements (Horn, 1982); (2) the haltere system, which detects rotations of the body and can induce adequate compensatory head movements (Sandeman, 1980a; Sandeman, 1980b; Nalbach and Hengstenberg, 1994; Huston and Krapp, 2009); and (3) the prosternal organ on the ventral side of the fly's neck, which senses head rotations and posture and initiates compensatory head movements (Horn, 1982; Strausfeld and Seyan, 1982; Hengstenberg, 1993; Paulk and Gilbert, 2006; Sandeman, 1980a).

At first sight, our results seem to differ from earlier findings on head and body movements of free-walking blowflies (Blaj and van Hateren, 2004), where virtually no head and body rotations were observed during straight walking phases, but were found to be mainly 
due to yaw saccades. These differences can be explained by different experimental paradigms. On the one hand, Blaj and van Hateren (Blaj and van Hateren, 2004) were not able to record leg movements and, thus, did not have access to the walking cycle as a reference. On the other hand, the blowflies in their experiments walked freely on a plain surface and revealed a saccadic walking mode by changing frequently their direction (Blaj and van Hateren, 2004). During the straight intersaccadic segments, the flies walked with velocities between 0 and $4 \mathrm{~cm} \mathrm{~s}^{-1}$, i.e. considerably slower than in our experiments. Based on the relationship between walking speed and stride frequency as observed in the present study, the stride duration at walking velocities as small as those observed by Blaj and van Hateren (Blaj and van Hateren, 2004) is predicted to be well above $100 \mathrm{~ms}$. This suggests that for intersaccadic intervals of, on average, less than $100 \mathrm{~ms}$, as estimated on the basis of the data of Blaj and van Hateren (Blaj and van Hateren, 2004), not even one complete stride cycle takes place between consecutive saccades under these experimental conditions. In addition, the walking pattern might deviate from a regular tripod gait, as has been reported for slowly walking Drosophila (Strauss and Heisenberg, 1990). We assume that the lower rotational head and body fluctuations recorded by Blaj and van Hateren (Blaj and van Hateren, 2004) might be due to the specific features of the intersaccadic stride pattern at slow walking speeds.

To compare the stabilization performance of the head during walking and during flight, we took flight data of van Hateren and Schilstra (van Hateren and Schilstra, 1999a; van Hateren and Schilstra, 1999b) and analyzed the rotational velocities during 81 intersaccadic flight sections which were at least $60 \mathrm{~ms}$ long and, thus, were at least about as long as the average length of one stride cycle, i.e. $67.5 \mathrm{~ms}$. The head stability in intersaccadic flight is considerably better than during straight walking on flat ground. Yaw fluctuations are six times smaller and pitch and roll rotations are approximately three times smaller (mean head fluctuations in intersaccadic flight: yaw, $0.65 \pm 0.3 \mathrm{deg}$; pitch, $0.86 \pm 0.1 \mathrm{deg}$; roll, $0.84 \pm 0.4 \mathrm{deg}$ ). Moreover, mean rotational head velocities are approximately 2.5 times slower in straight flight compared with straight walking on flat ground (mean head rotation velocities in intersaccadic flight: yaw, $61.8 \pm 8 \mathrm{deg} \mathrm{s}^{-1}$; pitch, $78.85 \pm 7 \mathrm{deg} \mathrm{s}^{-1}$; roll, 93.4 $\pm 21 \mathrm{deg} \mathrm{s}^{-1}$ ). Obviously, the walking apparatus, even on a flat substrate, causes larger head fluctuations than the flight apparatus during the straight intersaccadic intervals. This difference should be caused mainly by the extent of body fluctuations both types of locomotion produce by interacting with the particular medium. The impact of this interaction during walking on a generally solid medium is stronger and more direct than the impact during flight through less solid, viscous air and, therefore, may produce more rotational body fluctuations.

To what extent do the head fluctuations during walking affect visual information processing? The first processing stages in insect motion vision can be explained by the model of elementary motion detection (Egelhaaf and Reichardt, 1987; Borst and Egelhaaf, 1989; Egelhaaf and Borst, 1989; Hassenstein and Reichardt, 1956), in which the visual input from neighboring photoreceptors or ommatidia is processed in a directionally selective way. The interommatidial angles in the blowfly's compound eye range between $1.2 \mathrm{deg}$ in the frontal visual field and $3 \mathrm{deg}$ in the dorsal pole region (Petrowitz et al., 2000). Hence, the rotational head fluctuations of approximately $4 \mathrm{deg}$ induce rotational image slip (optic flow) that extends across one to three neighboring ommatidia, and thus should be detectable by the visual system of the fly.

Elegant studies have shown that flying as well as walking insects perform saccadic gaze strategies to support spatial information acquisition from the optic flow generated by their self-motion (Schilstra and van Hateren, 1998; Blaj and van Hateren, 2004; Boeddeker et al., 2010). To enable the fly's visual system to extract spatial information effectively from the optic flow generated during walking, the rotational optic flow components should be considerably smaller than the translational components. Translational optic flow is strong for close objects and fast movement speeds and weak for more distant objects and slow movements. Because flies move slower during walking than during flight, the spatial range in which translational flow dominates and the fly is able to obtain spatial information is smaller during walking than during flight. Furthermore, the rotational head fluctuations during straight walking cause additional rotational optic flow, which further reduces the distance range in which translational flow prevails. Consequently, the range in which walking flies can extract distance information is expected to be significantly reduced compared with flight.

How far-reaching is distance estimation by optic flow information during walking? One possible approach to investigate this issue may be to record the activity of optic-flow-processing neurons during stimulation with reconstructed optic flow as perceived by freely walking blowflies, as has already been done for flying flies (Kern et al., 2006; Karmeier et al., 2006). Because behavioral activity and, in particular, locomotion can affect the response properties of these neurons to some extent (Jung et al., 2011; Chiappe et al., 2010; Maimon et al., 2010; Rosner et al., 2010), it would be desirable to make neuronal recordings in the behaving fly as has recently been successfully accomplished in the much larger locust (Fotowat et al., 2011). With this new technique, it might be possible to estimate what spatial information can be extracted from optic-flow-processing neurons during free walking.

\section{ACKNOWLEDGEMENTS}

We thank R. Kern and N. Boedekker for critical comments on the manuscript, E. Braun and J. P. Lindemann for fundamental help in establishing the head and body tracking technique, and T. Siesenop for his help with data acquisition. Furthermore, we thank D. Schwyn, H. Krapp (Imperial College, London) and the Natural History Museum, London, for kindly providing the 3-D model of the head of the blowfly Calliphora vicina, as well as M. Botsch (Computer Graphics and Geometry Processing, Bielefeld University) for reducing the polygon complexity of the 3-D model.

\section{FUNDING}

This study was supported by the Human Frontier Science Program (HFSP) [RPG16/2008] and the Deutsche Forschungsgemeinschaft (DFG).

\section{REFERENCES}

Blaj, G. and van Hateren, J. H. (2004). Saccadic head and thorax movements in freely walking blowflies. J. Comp. Physiol. A 190, 861-868.

Boeddeker, N. and Hemmi, J. M. (2010). Visual gaze control during peering flight manoeuvres in honeybees. Proc. R. Soc. Lond. B 277, 1209-1217.

Boeddeker, N., Dittmar, L., Stürzl, W. and Egelhaaf, M. (2010). The fine structure of honeybee head and body yaw movements in a homing task. Proc. R. Soc. Lond. B 277, 1899-1906.

Borst, A. and Egelhaaf, M. (1989). Principles of visual motion detection. Trends Neurosci. 12, 297-306.

Borst, A., Haag, J. and Reiff, D. F. (2010). Fly motion vision. Annu. Rev. Neurosci. 33, $49-70$.

Bouguet, J. Y. and Perona, P. (1998). Camera Calibration From Points and Lines in Dual-space Geometry. Technical report. Pasadena, CA: California Institute of Technology.

Chiappe, M. E., Seelig, J. D., Reiser, M. B. and Jayaraman, V. (2010). Walking modulates speed sensitivity in Drosophila motion vision. Curr. Biol. 20, 1470-1475.

Dethier, V. G. (1976). The Hungry Fly: a Physiological Study of the Behavior Associated with Feeding. Cambridge, MA: Harvard University Press.

Dunlap, K. and Mowrer, O. H. (1930). Head movements and eye functions of birds. J. Comp. Psychol. 11, 99-113.

Egelhaaf, M. (2006). The neural computation of visual motion information. In Invertebrate Vision (ed. E. Warrant and D. E. Nilsson), pp. 399-461. Cambridge: Cambridge University Press.

Egelhaaf, M. and Borst, A. (1989). Transient and steady-state response properties of movement detectors. J. Opt. Soc. Am. A 6, 116-127. 
Egelhaaf, M. and Reichardt, W. (1987). Dynamic response properties of movement detectors: theoretical analysis and electrophysiological investigation in the visual system of the fly. Biol. Cybern. 56, 69-87.

Fotowat, H., Harrison, R. R. and Gabbiani, F. (2011). Multiplexing of motor information in the discharge of a collision detecting neuron during escape behaviors. Neuron 69, 147-158.

Geurten, B. R. H., Kern, R., Braun, E. and Egelhaaf, M. (2010). A syntax of hoverfly flight prototypes. J. Exp. Biol. 213, 2461-2475.

Grossman, G., Leigh, R., Abel, L., Lanska, D. and Thurston, S. (1988). Frequency and velocity of rotational head perturbations during locomotion. Exp. Brain Res. 70, 470-476.

Hardie, R. C. (1979). Electrophysiological analysis of fly retina. I: Comparative properties of R1-6 and R7 and 8. J. Comp. Physiol. A 129, $19-33$.

Hassenstein, B. and Reichardt, W. (1956). Systemtheoretoische Analyse der ZeitReihenfolgen- und Vorzeichenauswertung bei der Bewegungsperzeption des Rüsselkäfers Chlorophanus. Z. Naturforschg. 11, $513-524$.

Hengstenberg, R. (1986). Compensatory head roll in the blowfly Calliphora during flight. Proc. R. Soc. Lond. B 227, 455-482.

Hengstenberg, R. (1991). Gaze control in the blowfly Calliphora: a multisensory, twostage integration process. Semin. Neurosci. 3, 19-29.

Hengstenberg, R. (1993). Multisensory control in insect oculomotor systems. Rev. Oculomot. Res. 5, 285-298.

Hirasaki, E., Moore, S. T., Raphan, T. and Cohen, B. (1999). Effects of walking velocity on vertical head and body movements during locomotion. Exp. Brain Res. 127, 117-130.

Horn, E. (1982). Gravity reception in the walking fly, Calliphora erythrocephala: tonic and modulatory influences of leg afferents on the head position. J. Insect Physiol. 28, 713-721.

Horn, E. and Lang, H.-G. (1978). Positional head reflexes and the role of the prosternal organ in the walking fly, Calliphora erythrocephala. J. Comp. Physiol. A 126, 137-146.

Horn, E. and Mittag, J. (1980). Body movements and retinal pattern displacements while approaching a stationary object in the walking fly, Calliphora erythrocephala. Biol. Cybern. 77, 67-77.

Huston, S. J. and Krapp, H. G. (2008). Visuomotor transformation in the fly gaze stabilization system. PLoS Biol. 6, e173.

Huston, S. J. and Krapp, H. G. (2009). Nonlinear integration of visual and haltere inputs in fly neck motor neurons. J. Neurosci. 29, 13097-13105.

Jung, S. N., Borst, A. and Haag, J. (2011). Flight activity alters velocity tuning of fly motion-sensitive neurons. J. Neurosci. 31, 9231-9237.

Karmeier, K., van Hateren, J. H., Kern, R. and Egelhaaf, M. (2006). Encoding of naturalistic optic flow by a population of blowfly motion-sensitive neurons. $J$. Neurophysiol. 96, 1602-1614.

Kemmerling, S. and Varju, D. (1981). Regulation of the body-substrate-distance in the stick insect: responses to sinusoidal stimulation. Biol. Cybern. 137, 129-137.

Kern, R., van Hateren, J. H. and Egelhaaf, M. (2006). Representation of behaviourally relevant information by blowfly motion-sensitive visual interneurons requires precise compensatory head movements. J. Exp. Biol. 209, 1251-1260.

Land, M. F. (1973). Head movements of flies during visually guided flight. Nature 243 299-300.

Land, M. F. (1999). Motion and vision: why animals move their eyes. J. Comp. Physiol. A 185, 341-352.

Maimon, G., Straw, A. D. and Dickinson, M. H. (2010). Active flight increases the gain of visual motion processing in Drosophila. Nat. Neurosci. 13, 393-399.
McNeill, R. A. (2003). Principles of Animal Locomotion. Princeton, NJ: Princeton University Press.

Milde, J. J., Seyan, H. S., and Strausfeld, N. J. (1987). The neck motor system of the fly Calliphora erythrocephala. II. Sensory organization. J. Comp. Physiol. A 160 205-238.

Miles, F. A. (1997). Visual stabilization of the eyes in primates. Curr. Opin. Neurobiol. 7, 867-871.

Nalbach, G. and Hengstenberg, R. (1994). The halteres of the blowfly Calliphora. J. Comp. Physiol. A 175, 695-708.

Necker, R. (2007). Head-bobbing of walking birds. J. Comp. Physiol. A 193, 11771183.

Paulk, A. and Gilbert, C. (2006). Proprioceptive encoding of head position in the black soldier fly, Hermetia illucens (L.) (Stratiomyidae). J. Exp. Biol. 209, 3913-3924.

Petrowitz, R., Dahmen, H., Egelhaaf, M. and Krapp, H. G. (2000). Arrangement of optical axes and spatial resolution in the compound eye of the female blowfly Calliphora. J. Comp. Physiol. A 186, 737-746.

Pozzo, T., Berthoz, A. and Lefort, L. (1990). Head stabilization during various locomotor tasks in humans. Exp. Brain Res. 82, 97-106.

Riggs, L. A., Berry, R. N. and Wayner, M. (1949). A comparison of electrical and psychophysical determinations of the spectral sensitivity of the human eye. J. Opt. Soc. Am. 39, 427-436.

Rosner, R., Egelhaaf, M and Warzecha, A.-K. (2010). Behavioural state affects motion-sensitive neurones in the fly visual system. J. Exp. Biol. 213, 331-338.

Sandeman, D. C. (1980a). Head movements in flies (Calliphora) produced by deflexion of the halteres. J. Exp. Biol. 85, 43-60.

Sandeman, D. C. (1980b). Angular acceleration, compensatory head movements and the halteres of flies (Lucilia serricata). J. Comp. Physiol. A 136, 361-367.

Schilstra, C. and van Hateren, J. H. (1998). Stabilizing gaze in flying blowflies. Nature 395, 654

Schwyn, D. A., Heras, F. J. H., Bolliger, G., Parsons, M. M., Krapp, H. G. and Tanaka, R. J. (2011). Interplay between feedback and feedforward control in fly gaze stabilization. 18th IFAC World Congress Milano, August 28-September 2, 2011, 9674-9679.

Strausfeld, N. J. and Seyan, H. S. (1982). Convergence of visual, haltere, and prosternal inputs at neck motor neurons of Calliphora erythmocephala. Cell Tissue Res. 240, 601-615.

Strauss, R. and Heisenberg, M. (1990). Coordination of legs during straight walking and turning in Drosophila melanogaster. J. Comp. Physiol. A 167, 403-412.

van Hateren, J. H. and Schilstra, C. (1999a). Blowfly flight and optic flow. I. Thorax kinematics and flight dynamics. J. Exp. Biol. 202, 1481-1490.

van Hateren, J. H. and Schilstra, C. (1999b). Blowfly flight and optic flow. II. Head movements during flight. J. Exp. Biol. 202, 1491-1500.

Waespe, W. and Henn, V. (1987). Gaze stabilization in the primate. In Reviews of Physiology, Biochemistry and Pharmacology, Vol. 106 (ed. O. Krayer, E. Lehnartz, A. Muralt and H. H. Weber), pp. 37-125. Heidelberg: Springer.

Wagner, H. (1986). Flight performance and visual control of flight of the free-flying housefly (Musca domestica L.). I. Organization of the flight motor. Philos. Trans. R. Soc. Lond. B 312, 527-551.

Wallmann, J. and Letelier, J. C. (1993). Eye movements, head movements, and gaze stabilization in birds. In Vision, Brain, and Behavior in Birds (ed. H. P. Zeigler and H. J. Bischof), p. 415. Cambridge, MA: MIT Press.

Zeigler, H. P. and Bischof, H. J. (1993). Vision, Brain and Behavior in Birds. Cambridge, MA: MIT Press. 